Ophthalmologe 2009 · 106:193-193

DOI 10.1007/s00347-008-1903-z

Online publiziert: 28. Februar 2009

(c) Springer Medizin Verlag 2009
H.-W. Meyer-Rüsenberg ${ }^{1}$ K.-H. Emmerich ${ }^{2}$

${ }^{1}$ Augenklinik, St. Josefs-Hospital, Universität Witten-Herdecke, Hagen

${ }^{2}$ Augenklinik, Klinikum Darmstadt

\title{
Therapieoptionen bei Tränenwegserkrankungen
}

Das tränende Auge mit dem Leitsymptom Epiphora ist für die Betroffenen mit einer hohen, manchmal unerträglichen Belastung verbunden. Ursache des tränenden Auges können verschiedene Faktoren sein: Hypersekretion, Tränenfilmstörungen, Störungen der Tränenpumpfunktion (beispielsweise durch Lidfehlstellungen und Verletzungen) oder mechanische Hindernisse.

Mechanische Hindernisse führen früher oder später immer zu entzündlichen Veränderungen wie einer Dakryozystitis oder einer Dakryophlegmone. Seit der Antike gibt es Beschreibungen von teilweise heroischen Operationsverfahren zur Beseitigung dieser entzündlichen Veränderungen. Doch beginnt die erfolgreiche Therapie unter weitgehendem Erhalt der Funktion erst mit der Beschreibung der externen Dakryozystorhinostomie (DCR) durch Toti und der internen endonasalen DCR nach West. Nach einer Vielzahl von Modifikationen dieser Verfahren ermöglicht erst der Einsatz von Endoskopen und Mikroendoskopen sowie die Verwendung von Lasern eine Verbesserung der Ergebnisse und eine Verminderung der Belastung für die betroffenen Patienten.

Die vorliegenden Arbeiten geben einen aktuellen Überblick über den heutigen Stand der unterschiedlichen Aspekte der Tränenwegchirurgie. Die DCR in ihren beiden konkurrierenden Zugangswegen, extern oder intern, ist nach wie vor nicht vollständig ersetzbar. Die ungeheure Vielzahl an Publikationen über die DCR unter Verwendung unterschiedlichster Hilfsmittel belegt, dass unabhängig vom Grundprinzip der DCR (Schaffung einer neuen Verbindung von Tränensack und Nase) kein Königsweg besteht. Die nicht exakte Definition von Ausgangsbefunden und Erfolgskriterien erschwert die Vergleichbarkeit der verschiedenen Studien oder macht Vergleiche sogar unmöglich.

Die Tränenwege liegen in unmittelbarer Nachbarschaft der Nasenhaupt- und Nasennebenhöhlen. In der Arbeit von Bloching und Prescher wird die HNOärztliche Sicht bei Erkrankungen der ableitenden Tränenwege dargestellt. Auch diese Ergebnisse zeigen, wie unerlässlich die interdisziplinäre Zusammenarbeit von Augenarzt und Hals-Nasen-Ohren-Arzt bei Erkrankungen der ableitenden Tränenwege ist.

Im Weiteren werden die Entwicklung und der Stellenwert der transkanalikulären Mikroendoskopie und der daraus hervorgegangenen minimal-invasiven Tränenwegchirurgie beschrieben. Hierbei handelt es sich um die einzige Methode in der Behandlung mechanischer Tränenwegstenosen, bei der die anatomische und physiologische Integrität des tränenableitenden Systems mit der Muskelpumpe erhalten bleibt.

Tränenwegverletzungen erfordern eine sachgerechte und zeitnahe Primärversorgung. Das Know-how hierfür muss daher an jeder Klinik der Maximalversorgung abrufbar sein. Epidemiologie sowie Grundprinzipien der Wiederherstellungschirurgie und deren Erfolgsraten werden von Struck erläutert.

Störungen der Tränenpumpe und damit die funktionellen Aspekte einer symptomatischen Epiphora werden in der Arbeit von Schaudig detailliert vorgestellt. Die Ursachen von Epiphora bei mechanisch durchgängigen Tränenwegen, v. a. im höheren Lebensalter, werden anschaulich erklärt.
Tränenfilmstörungen gehören ebenfalls in Diagnostik und Therapie zum Gesamtkomplex Epiphora und sind in den letzten Jahren intensiv erforscht worden. Der aktuelle Stand dieser Forschungsergebnisse wird dargestellt und therapeutische Möglichkeiten werden aufgezeigt.

Wir wünschen Ihnen mit den vorliegenden Artikeln zu dem Schwerpunktthema und den anderen Beiträgen dieser Ausgabe von „Der Ophthalmologe“ eine gewinnbringende Lektüre und vielfältige Anregungen.

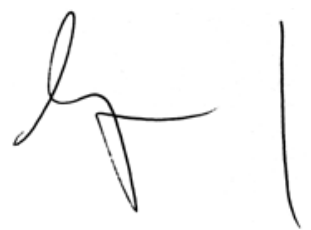

H.-W. Meyer-Rüsenberg

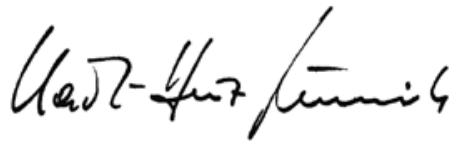

K.-H. Emmerich

Korrespondenzadresse

Prof. Dr. H.-W. Meyer-Rüsenberg

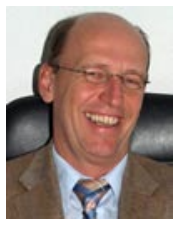
Augenklinik, St. Josefs-Hospital, Universität Witten-Herdecke 58097 Hagen meyer-ruesenberg@ kkh-hagen.de

Prof. Dr. K.-H. Emmerich

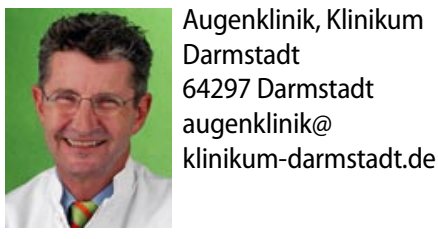

